Dr CAVAlier-Smith RePlies-I like Bateman's modification of my model. It implies that a non-replicating eukaryote chromosome is a single, circular selfcomplementary polynucleotide chain; there are several ways this might be tested. In fact recent evidence indicates that vaccinia virus DNA may have this structure $^{1}$. Some other viruses do not, however, and an enzyme system has been isolated ${ }^{2}$ from phage $\mathrm{T} 7$ which can crosslink the termini of some of them (T7, $\mathrm{T} 3, \mathrm{~T} 1$ and $\lambda$ ) in the ways postulated in my original paper ${ }^{3}$.

This implies that even these phages have terminal palindromes; they may therefore be able to replicate according to my original model as well as (or instead of) Watson's concatomeric one. Another model involving a terminal palindrome has been independently proposed $^{4}$ for the replication of linear mitochondrial DNA in Tetrahymena, But this postulates a palindrome only at one end and therefore requires a circular intermediate, and so seems implausible for eukaryote nuclear DNA.

Department of Biophysics, School of Biological Sciences,

University of London King's College, 26-29 Drury Lane, London WC2B 5RL, UK

${ }^{1}$ Geshelin, P., and Berns, K. I., J. molec. Biol., 88, 785-796 (1974).

2 Sadowski, P., McGeer, A., and Becker, A., Can. J. Biochem., 52, 525-535 (1974).

${ }^{3}$ Cavalier-Smith, T., Nature, 250, 467-470 (1974).

${ }^{4}$ Arnberg, A. C., Van Bruggen, E. F. J., Clegg, R. A., Upholt, W. P., and Borst, P., Biochim. biophys. Acta, 361, 266276 (1974).

\section{Hepatocyte sialoglycoprotein}

EvANs $^{1}$ reported that the plasma membrane enzyme nucleotide pyrophosphatase is located on the external face of the hepatocyte surface. Based on this result, Evans argues that proposals which suggest cell surface glycosyl transferases function in cell adhesion ${ }^{2,3}$ and in selective binding of asialo-glycoproteins ${ }^{4}$ are less tenable.

As nucleotide pyrophosphatase on the outer membrane surface rapidly cleaves nucleotide sugars and Roseman ${ }^{2}$ has placed an important role for these glycosyl transferase substrates in his theory of cell adhesion, Evans says the proposed mechanism would not be possible. I wish, however, to re-emphasise our results which implicate liver plasma membrane galactosyl transferase in the binding of asialo-glycoproteins ${ }^{4}$. We suggest that this result modifies Roseman's original proposal to the extent that cells remain attached to one another by complexes between glycosyl trans- ferases and their oligosaccharide products. Thus, the liver recognition of non-reducing terminal galactosyl residues on asialo-glycoproteins does not require any involvement of nucleotide sugars, as the active transferase seems to be galactosyl transferase for which the asialoprotein is a product, and not a substrate.

Evans' result suggests to us that another possible importance for nucleotide pyrophosphatase being localised on the exterior of hepatocytes is that this enzyme may catalyse degradation of external RNA. Clearly a cell must exclude foreign messenger RNA from its cytoplasmic environment, as such a molecule might function in protein synthesis resulting in the eventual transformation of that cell. Such a potent effect of RNA has already been shown to be operative for RNA tumour viruses. Naked RNA itself can be infective ${ }^{5}$, and the addition of other macromolecules which may complex with the RNA and thus protect it from enzymatic hydrolysis enhances infectivity ${ }^{6}$. Results have shown ${ }^{7-9}$ that liver plasma membrane degrades RNA, and inhibition of membrane phosphodiesterase (nucleotide pyrophosphatase) with EDTA diminishes this activity ${ }^{7,9}$. A second, well-established marker enzyme for the plasma membrane is $5^{\prime}$-nucleotidase and it also has been shown to be on the outside surface of both liver ${ }^{10}$ and leukocyte ${ }^{11}$ membranes. The $5^{\prime}$-nucleotide products of nucleotide pyrophosphatase would be substrates for $5^{\prime}$-nucleotidase, and indeed liver plasma membrane completely hydrolyses RNA to nucleosides plus inorganic phosphate ${ }^{7}$.

Nathan N. Aronson, Jun.

Department of Biochemistry,

The Pennsylvania State University,

University Park, Pennsylvania 16802

1 Evans, W. H., Nature, 250, 391 (1974).

2 Roseman, S., Chem. phys. Lipids, 5, 270 (1970).

${ }^{3}$ Roth, S., Q. Rev. Biol., 48, 541 (1973).

4 Aronson, N. N., jun., Tan, L. Y., and Peters, B. P., Biochem. biophys. Res. Commun., 53, 112 (1973).

\section{Matters arising}

Matters Arising is meant as a vehicle for comment and discussion about papers that appear in Nature. The originator of a Matters Arising contribution should initially send his manuscript to the author of the original paper and both parties should, wherever possible, agree on what is to be submitted. Neither contribution nor reply (if one is necessary) should be longer than 300 words and the briefest of replies, to the effect that a point is taken, should be considered.
5 Gierer, A. and Schramm, G. S., Nature, 177, 702 (1956).

${ }^{6}$ Seljelid, R., Silverstein, S. C., and Cohn, Z. A., J. Cell Biol., 57, 484 (1973).

7 Yannarell, A. and Aronson, N. N., jun., Biochim. biophys. Acta, 311, 191 (1973).

${ }^{8}$ Emmelot, P., Bos, C. J., Benedetti, E. L., and Rumke, P. H., Biochim. biophys. Acta, 90, 126 (1964).

9 Prospero, T. D., Burge, M. L. E., Norris, K. A., Hinton, R. H., and Reid, E., Biochem. J., 132, 449 (1973).

${ }^{10}$ Farquhar, M. G., Bergeron, J. J. M., and Palade, G. E., J. Cell Biol., 60, 8 (1974).

11 DePierre, J. W., and Karnovsky, M. L., Science, 183, 1096 (1974).

Dr EvanS REPLIES-Aronson is correct in pointing out that the binding of asialoglycoproteins by a receptor, possibly a galactosyl transferase present in a liver plasma membrane fraction can occur without a requirement for sugar nucleotides. The involvement of sugar nucleotides in the transglycosylation reaction was postulated by Roseman to account for the de-adhesion process ${ }^{1}$ and therefore their hydrolysis by the surfacelocated nucleotide pyrophosphatase would be a problem. Such proposals, however, must remain tentative until it is demonstrated more clearly that galactosyl and other sugar transferases are indigenous and functional components of the liver plasma membrane, and do not reflect contamination by Golgi membranes.

The plasma membrane fraction used by Aronson was shown to derive mainly from the liver sinusoidal surface ${ }^{2}$ and to be contaminated by Golgi membranes as indicated by morphological markers and high activities of galactosyl and sialyl transferases $^{3.4}$ that we have shown to be absent from the contiguous and bile canalicular face subfractions ${ }^{3}$. A fuller assessment of a possible role for cell surface galactosyl transferases and associated enzymes in the binding of serum components and in cell adhesion must await further analysis of the specific domains of the surface membrane and their interrelationships with Golgi membranes. Recently, the absence of cell surface galactosyl transferase in a number of cell lines was reported ${ }^{5}$, supporting the general conclusion that this enzyme does not play a role in cellular adhesion, cell recognition and contact inhibition.

Medical Research Council,

National Institute for Medical Research, London $N W 7, U K$

' Roseman, S., Chem. Phys. Lipids, 5, 270-297 (1970).

${ }^{2}$ Wisher. M. H., and Evans, W. H., Biochem. Soc. Trans., 2, 407-408 (1974).

3 Wisher, M. H., and Evans, W. H., Biochem. J., (in the press).

+ Dewald, B. and Touster, O., J. biol Chem., 248, 7223-7233 (1973)

5 Deppert, W., Werschan, H., and Walter, G., Proc. natn. Acad. Sci. U.S.A., 71, 3068-3072 (1974). 